\title{
Computational Modeling of Large Wildfires: A Roadmap
}

\author{
Janice L. Coen \\ National Center for Atmospheric Research \\ NCAR Earth System Laboratory \\ P. O. Box 3000, Boulder, Colorado 80307 USA \\ e-mail: janicec@ucar.edu
}

\author{
Craig C. Douglas \\ School of Energy Resources and Mathematics Dept. \\ University of Wyoming \\ Laramie, Wyoming 82071-3036, USA \\ e-mail: craig.c.douglas@gmail.com
}

\begin{abstract}
Wildland fire behavior, particularly that of large, uncontrolled wildfires, has not been well understood or predicted. Our methodology to simulate this phenomenon uses high-resolution dynamic models made of numerical weather prediction (NWP) models coupled to fire behavior models to simulate fire behavior. NWP models are capable of modeling very high resolution $(<100 \mathrm{~m})$ atmospheric flows. The wildland fire component is based upon semi-empirical formulas for fireline rate of spread, post-frontal heat release, and a canopy fire. The fire behavior is coupled to the atmospheric model such that low level winds drive the spread of the surface fire, which in turn releases sensible heat, latent heat, and smoke fluxes into the lower atmosphere, feeding back to affect the winds directing the fire. These coupled dynamic models capture the rapid spread downwind, flank runs up canyons, bifurcations of the fire into two heads, and rough agreement in area, shape, and direction of spread at periods for which fire location data is available. Yet, intriguing computational science questions arise in applying such models in a predictive manner, including physical processes that span a vast range of scales, processes such as spotting that cannot be modeled deterministically, estimating the consequences of uncertainty, the efforts to steer simulations with field data ("data assimilation"), lingering issues with short term forecasting of weather that may show skill only on the order of a few hours, and the difficulty of gathering pertinent data for verification and initialization in a dangerous environment.
\end{abstract}

Keywords-Numerical model; weather prediction, wildland fire model, wildfire, forest fire, fire behavior

\section{INTRODUCTION}

Wildland fires present substantial scientific, computational, and forecasting challenges. Large, intense wildfires especially are difficult to understand and model because they combine complicated meteorology, spatially heterogeneous and physically complex fuel structures, and fire phenomena that are not present in smaller, less intense fires or reproducible in prescribed fires or laboratory experiments. Simulation of large wildfires is a computational challenge because of the vast range of spatial and temporal scales (perhaps 7 orders of magnitude from synoptic-scale weather at 10 's of $\mathrm{km}$ to centimeter-scale combustion processes) that contribute substantially to these nonlinear phenomena. Moreover, the fire line defining the interface between burning and unburned regions is a subgrid scale phenomena; treatments of moving interfaces within grid cells have developed into a field of study (e.g. particle-in-cell techniques, level set methods, etc.).

Forecasting wildfire growth involves all the complexity, errors, and uncertainty of weather prediction compounded by the uncertainty of modeling the fire process itself. Weather forecasting issues that are especially relevant to this application are (for multi-day strategic modeling) the limits of predictability, particularly the degradation of numerical weather prediction forecast skill with time, and for very short term forecasts, the very low skill of predicting convection (that is, the vertical current of air that transport heat from the surface upwards), the winds from which strongly influence surface winds directing fire behavior.

We will present results of landscape-scale wildland fire simulations to illustrate our current capabilities to model these phenomena using a coupled weather-wildland fire model, foremost for research and understanding but addressing questions posed as to their suitability as a predictive tool. These computer simulations use numerical weather prediction models tied to fire behavior models to simulate the impact of a fire on the atmosphere and the subsequent feedback of these fire-induced winds on fire behavior - i.e. how all fires, to some degree, 'create their own weather'. The methodology involves the use of a numerical weather prediction model capable of modeling fine scale atmospheric flows (under $1 \mathrm{~km}$ horizontal resolution) in very steep (slope where the rise over run of terrain may exceed 0.6 ) terrain. The wildland fire component is based upon semi-empirical relationships (one example being the Rothermel surface fire algorithms [1]) and a canopy fire model. The fire behavior is coupled to the atmospheric model such that low level winds drive the spread of the surface fire, which in turn release sensible heat, latent heat, and smoke fluxes into the lower atmosphere, in turn feeding back to affect the winds directing the fire. Although this influence is most dramatic near the fire, model simulations show this influence can change the wind speed by several kilometers per hour even 
kilometers from the fire. An extreme example of this feedback is the blowup known as a 'firestorm'.

We address the computational aspects of running such models in real time for forecasting of fire behavior in the current computing environment. Although the meteorological aspects of forecasting have been mentioned previously, the computational aspects of running such models in real time have not yet been examined. We will (1) introduce two different models created for this sort of study and discuss their limitations as predictions when applying them as forecasting tools, (2) address the challenges of modeling physical processes that span a vast range of scales, (3) discuss processes such as spotting that cannot be modeled deterministically, (4) discuss options for estimating the consequences of uncertainty, and (5) discuss the efforts to steer simulations with field data ("data assimilation") and the difficulty of gathering pertinent data for verification, initialization, and steering in a dangerous environment.

\section{MODEL DESCRIPTION}

A coupled weather-fire modeling system is composed of two parts: a numerical weather prediction model and a fire behavior model that models the growth of a wildfire in response to weather, fuel conditions, and terrain.

\section{A. Atmospheric Model}

In one implementation (CAWFE, the Coupled Atmosphere-Wildland Fire Environment model), the weather model ([2], [3]) is a small-scale atmospheric research model. In both cases, the weather and fire components are two-way coupled so that heat and water vapor fluxes from the fire alter the atmospheric state, notably producing fire winds, while the evolving atmospheric state and changes in humidity (including effects from the fire) in turn affect fire behavior, notably how fast and in what direction the fire propagates. This wildfire

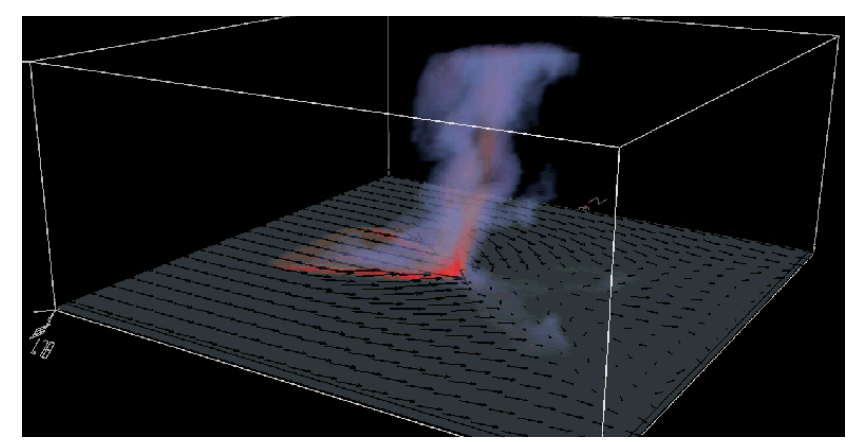

Figure 1. Simulation of a wildfire spreading in chaparral shrub in an environmental wind of $3 \mathrm{~m} \mathrm{~s}-1$ from the left. The vectors represent the near-surface wind, the misty purple field is the smoke concentration produced by the fire, and the red is the buoyancy (warm air) produced by the fire. simulation model can thus represent the complex interactions between a fire and the atmosphere.

The meteorological model is a three-dimensional nonhydrostatic numerical model based on the Navier-Stokes equations of motion, a thermodynamic equation, and conservation of mass equations using the anelastic approximation. Vertically stretched terrain-following coordinates allow the user to simulate in detail the airflow over complex terrain. Forecasted changes in the larger-scale atmospheric environment are used to initialize the outer of several nested domains and update lateral boundary conditions. Two-way interactive nested grids capture the outer forcing domain scale of the synoptic-scale environment while allowing the user to telescope down to tens of meters near the fireline through horizontal and vertical grid refinement. Weather processes such as the production of cloud droplets, rain, and ice and subgrid-scale eddy diffusion are parameterized.

In a second implementation, the weather model is the Weather Research and Forecasting model (WRF) [4], the new community model now being used at U.S. national forecasting centers. The WRF-Fire component has been released public domain and is available along with the WRF V3.2 on April 2, 2010 at wrf-model.org, or at the project website www.openwfm.org .

\section{B. Fire Model}

Local fire spread rates depend on the modeled wind components through an application of the semi-empirical Rothermel fire spread formula [1]. The heat release rate is based on [5] which characterizes how the fire consumes fuels of different sizes with time after ignition, distinguishing between rapidly consumed grasses and

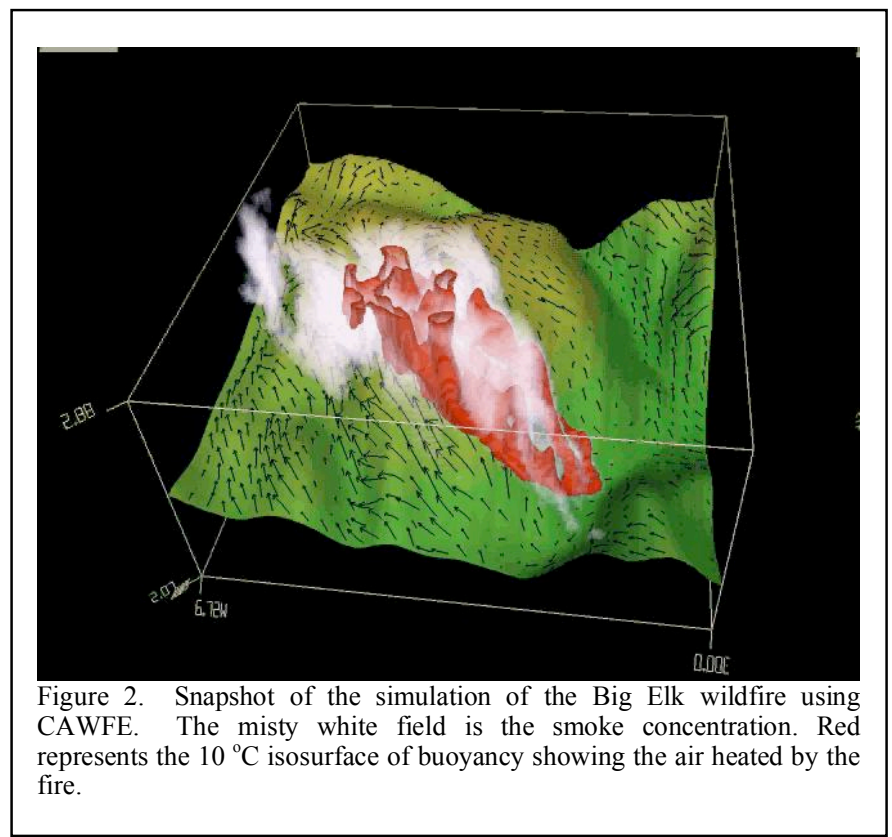


slowly burned logs. Within each atmospheric grid cell, the land surface is further divided into fuel cells with fuel characteristics corresponding to 13 standard fuel types [6].

In CAWFE, four moving points, called tracers, assigned to each fuel cell, identify burning areas of fuel cells and define the fire front. Within each cell on the fire model grid, a quadrilateral defines the burning region. Fire spread rates are calculated locally along the fire as a function of fuels, wind speed and direction from the atmospheric model (which includes the effects of the fire), and terrain slope, while a local contour advection scheme assures consistency along the fireline. The canopy may be dried and ignited by the surface fire. Then a simple radiation treatment distributes the sensible and latent heat into the lowest atmospheric grid levels.

This representation makes the fire area hard to adjust in data assimilation. For this reason, in WRF-Fire we have developed a translation of the tracers into a level function. The level function is given by values at nodes of the fire grid. The fire region is where the level function is positive. The absolute value of the level function is approximately equal to the Euclidean distance from the fireline. In data assimilation, the level function can be increased or decreased just like the physical quantities in the model.

\section{RESULTS}

A simple theoretical simulation is shown in Fig. 1 that simulates a rapidly spreading fireline in chaparral brush as it spreads into the commonly observed Universal Fire Shape.

Fig. 2 shows a snapshot of a simulation of the Big Elk fire, a wildland fire ignited in a mountain valley, as it

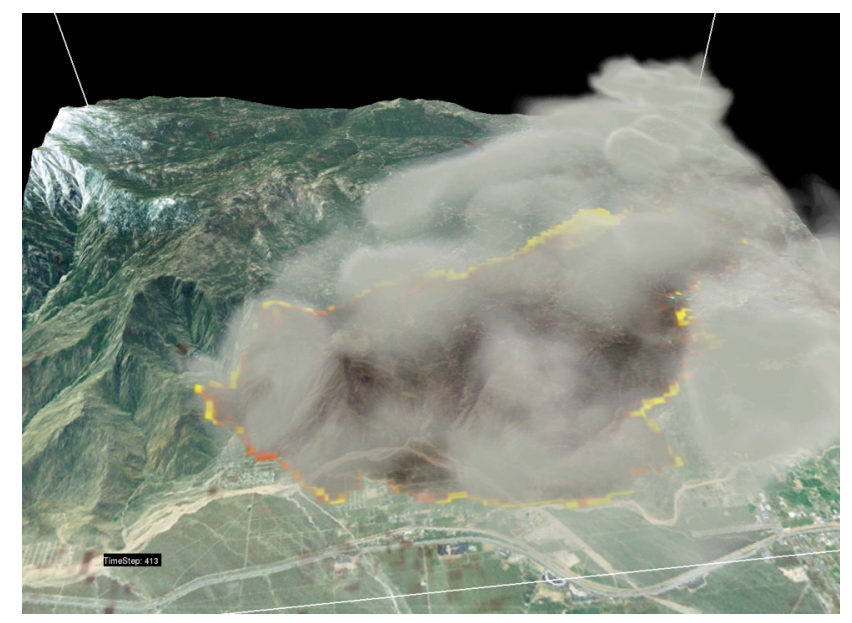

Figure 3. Simulation of the Esperanza wildfire at a time $3 \mathrm{hrs}$ before Figure 4. The gray field shows the smoke produced by the active regions of the fire (more intense regions in bright yellow, less intense in medium red, low intensity in dark red), where the active regions, as in Fig. 4., are only along the side flanks.

spreads up a mountain.

As an example of a large wildfire simulation using CAWFE, we address the Esperanza Wildfire, an arson- caused wildfire that ignited on October 26, 2006 at 0112 in a river wash outside Cabazon, CA. After ignition, it burned uphill driven by strong Santa Ana winds and steep slopes to the southwest. Spread was rapid due to strong winds (6-10 mph, 20-25 mph gusts), low relative humidity (6\%), and flammable chaparral brush. The atmospheric component of CAWFE was initialized with a gridded weather forecast for the region to examine the meteorological flow in the vicinity of the strong down slope wind-driven wildfire, model the fire growth and interaction with the atmospheric flow, and compare with measurements collected during the first few days of the fire. The model results (ex. In Fig. 3) capture the rapid spread downwind, flank runs up canyons, bifurcations of the fire into two heads, and rough agreement in area, shape, and direction of spread at periods for which fire location data is available. Secondly, results show that the acceleration of winds near the surface can be understood as resulting from complex three-dimensional atmospheric wave dynamics, contradicting the capping/squeezing mechanism stated in the fatality report - such simulations can be used recreate a physically consistent portrait of what happened. The extent of the fire as shown by aircraft infrared imagery at $11 \mathrm{am}$ (Fig. 4).

\section{DISCUSSION}

We have raised numerous issues about the practical application of a specific type of research model and not solved them. This area has many opportunities for students to apply themselves to. As mentioned earlier, although the meteorological aspects of forecasting have been mentioned previously, the computational aspects of running such models in real time have not yet been laid out. We believe it is beneficial to examine these issues in the context of the current computing environment. In the 1970's to 1990's, first

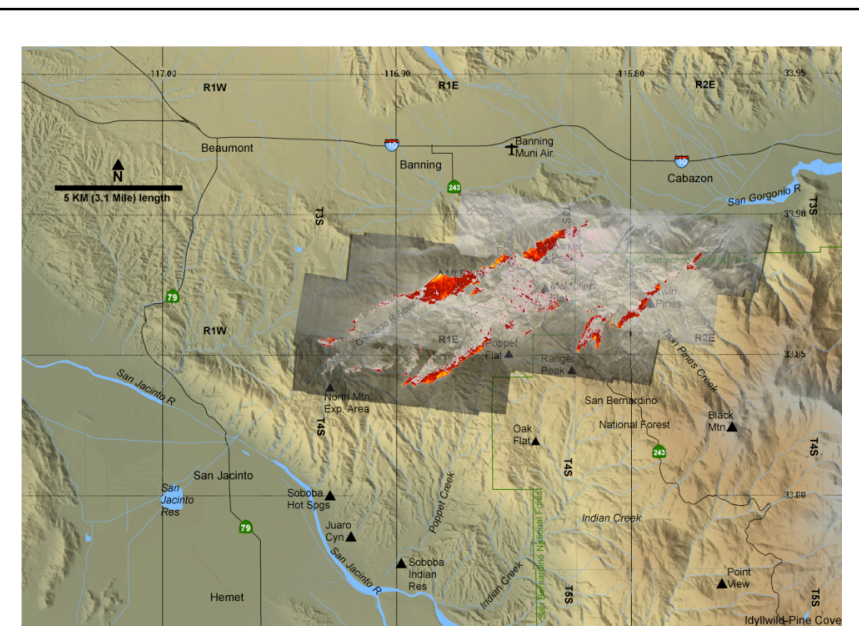

Figure 4. Sample image of radiant temperature from the airborne FireMapper infrared imager at 1117 local time during the Esperanza Wildfire. Image courtesy of Phillip Riggan, U.S.D.A. Forest Service. Bright oranges represent hotter temperatures, corresponding to more intensely burning regions of the fire. 
a vector supercomputer and later a shared memory supercomputer was necessary to solve wildland fire simulations in a reasonable amount of wall clock time. Today a desktop computer (or a blade in a rack server) is all that is necessary using the latest in multiple CPU, multicore shared memory architectures. For example, in 2010 using 4 CPUs with either 12 or 16 cores, we can compute with 48 or 64 cores conveniently using OpenMP and/or MPI for the parallel computing middleware.

We introduced two models created for this sort of study and discuss their limitations as predictions when applying them as forecasting tools. As the simulations show, there are many challenges to modeling physical processes that span a vast range of scales. The scales over which these simulation must cover are formidable. The synoptic (horizontal scale 1.e-5 km) and mesoscale (horizontal scale 1.e-3 $\mathrm{km}$ ) weather features must be included to provide the background wind, thermodynamic structure of the atmosphere, and wind. At the same time, fine resolution particularly near the surface is required to resolve the eddies providing the vertical heat fluxes of the fire model. Measurements from field observations suggest that even deep, energetic crown fires have profiles of vertical heat flux for which the e-folding depth is $50 \mathrm{~m}$, meaning that from a numerical methods perspective, several vertical grid points must be within this depth to resolve it. From a computational design perspective, this requires vertical grid refinement in inner domains, something that is not common in NWP models.

Moreover, some processes cannot be modeled deterministically. In a process called "spotting", wildfires release burning embers that are lofted in the fire plume and dropped from meters to many kilometers ahead of the fire in fuel, some of which ignite. Many variables including fuel moisture, the size distribution of the embers created, how high they rise and how much turbulence causes them to disperse, whether they are still burning when they reach the ground, the fuel in which they land and how moist it is, whether the fire has overtaken the landing spot, etc., factor in to whether and where these spot fires ignite. These can cause important bifurcations in a fire's progression, depending on whether they have spotted across a road or obstruction to the fire. It is possible to imagine stochastic models that consider a range of possible occurrences and their outcomes.

Further issues arise regarding the consequences of uncertainty. Due to the nonlinear nature of the phenomena involved and errors in our initial conditions, changes in initial conditions can cause wide divergence in model outcomes. Meteorologists often try to assess their certainty in an outcome by conducting a suite or 'ensemble' of simulations, basing their degree of confidence of the correctness of the outcome on the spread of outcomes. This desire has been formalized in the Ensemble Kalman method [7], assuming that the model error distribution is Gaussian and combining a Bayesian update of the model state with the model time step to incorporate data at times throughout the simulation. There are parts of the simulation that do parallelize well, e.g., linear algebra, discretization, and problem generation, but if we do not want to reprogram dusty deck software to be parallel (even mildly), we simply use an Ensemble Kalman filter and run 48-64 serial models with almost perfect parallel scaling. In fact, the scaling is so good that there is almost a disincentive to do small-scale parallelization. Moreover, the real-time problems posed by meteorologists are often not efficiently able to use more than a few hundred processors, each processor being reduced to a few grid points, beyond which communication costs overwhelm scaling efficiency.

Another interesting possibility is to consider hardware acceleration on general purpose, graphics processing units (GP-GPUs). In the codes that vectorize well, this option may be viable. However, due to the overall complexity and multiscale nature of wildland fire modeling, the cost of constantly loading, dumping, and reloading the contents of a GP-GPU's memory for the different scales currently takes too much time in comparison to just using 48-64 cores in the desktop (blade) computer.

Relevant data for initialization, validation, or assimilation could be weather data, possibly surface weather station data representing a still, time-evolving stream of information about the weather conditions at a point, or a weather sounding which is a vertical profile of the state of the atmosphere (pressure, temperature, moisture, and wind speed and direction) at one time. Radars or lidars may provide a 2- or 3-dimensional picture of the atmosphere, primarily the presence of clouds and precipitation and possibly, with some analysis, the winds. They can detect smoke from fires [8]. Much work in atmospheric science has concentrated on how to assimilate such commonly available data (surface data and vertical profiles are available over the web, as is processed radar data). An important assumption allowing that to occur is that the errors can be assumed to be distributed in a Gaussian profile. Some examples of assimilation of weather data are available [9] even to some extent with surface data [10], however, for remote locations where weather stations are sparse, assimilation may have no improvement on the weather component of a forecast of combined fire and weather.

Data can also be gathered on the fire itself, though this is not done routinely. As in Fig. 4, research flights and incident management teams may provide perimeters describing the extent of the fire. However, the bigger challenge and opportunity is to assimilate not just the perimeter, but also a field more quantitative and insightful and dynamic such as the spatial distribution of the radiant flux density from the fire map, which intuitively corresponds to the intensity of a burning fire. New techniques are being developed to assimilate this data [11] since the standard assumption of a Gaussian error distribution fails. Also, it is not simple to directly link 
radiance measurements or other field observations to some parameter in a model that can be steered with measurements (this function is known as the 'observation function').

Moreover, assimilating data disturbs the internal balance in the model and thus small perturbations are generally used particularly through the so-called nudging techniques, which nudge the simulation toward observed states.

While having data stream directly from the field will one day be an option, today most of the data has been collected during the fire and is used offline (i.e. not in real time) to validate models and improve firefighting strategies.

\section{ACKNOWLEDGMENT}

This research was supported in part by NSF grants 1018072, 1018079, 0324910 and 0835598 and Award No. KUS-C1-016-04, made by King Abdullah University of Science and Technology (KAUST). The National Center for Atmospheric Research is sponsored by the National Science Foundation. Any opinions, findings, and conclusions or recommendations expressed in this material are those of the authors and do not necessarily reflect the views of the National Science Foundation. Phillip J. Riggan, U.S.D.A. Forest Service, provided image 4.

\section{REFERENCES}

[1] R. C. Rothermel, "A mathematical model for predicting fire spread in wildland fuels". USDA Forest Service, Intermountain Forest and Range Experiment Station Research Paper INT-115, 1972. (Ogden, UT)
[2] T. L. Clark, J.L. Coen, D. Latham, "Description of a coupled atmosphere-fire model", Intl. Journal of Wildland Fire, vol. 13, 2004, pp. 49-63.

[3] J. L. Coen, "Simulation of the Big Elk Fire using using coupled atmosphere-fire modeling". Intl. J. Wildland Fire, vol. 14, 2005, pp. 49-59.

[4] WRF Working Group, 2008, "Weather Research and Forecasting (WRF) model" [Online]. http://www.wrfmodel.org

[5] F. A. Albini, "PROGRAM BURNUP: a simulation model of the burning of large woody natural fuels." Final Report on Research Grant INT-92754-GR by USFS to Montana State University, Mechanical Engineering Dept., 1994.

[6] H. E. Anderson, "Aids to determining fuel models for estimating fire behavior." USDA Forest Service, Intermountain Forest and Range Experiment Station Research Paper INT-122, 1982. (Ogden, UT).

[7] G. Evensen, "The ensemble Kalman filter: Theoretical formulation and practical implementation", Ocean Dynamics, vol. 53, pp. 343-367, 2003.

[8] J. Wurman, "Mobile Radars as a Fire Safety and Forecasting Tool", Proceedings, $9^{\text {th }}$ Wildland Fire Safety Summit, Pasadena, CA, 2006.

[9] E. Kalnay, Atmospheric Modeling, Data Assimilation and Predictability. Cambridge, U.K.: Cambridge Univ. Press, 2003.

[10] X. Deng and R. Stull, "Assimilating Surface Weather Observations from Complex Terrain into a High-Resolution Numerical Weather Prediction Model”. Mon. Wea. Rev., vol., 135, 2007, pp. 1037-1054.

[11] J. Mandel, L. S. Bennethum, J. D. Beezley, J. L. Coen, C. C. Douglas, M. Kim, and A. Vodacek, "A wildland fire model with data assimilation,” Math. Comput. Simul., vol. 79, 2008, pp. 584-606. 